INVESTIGACIÓN/RESEARCH

\title{
OBJETIVOS PEDAGÓGICOS BÁSICOS EN LA ENSEÑANZA DE LA ÉTICA DE LA COMUNICACIÓN
}

Jesús Díaz del Campo Lozano1: Universidad Internacional de La Rioja (UNIR). España. jesus.diaz@unir.net

\section{RESUMEN}

¿Cuáles son los objetivos pedagógicos básicos que debe perseguir un profesor de Ética de la Comunicación? En este artículo se analizan las respuestas más comunes dadas a esta cuestión por la bibliografía: Entender la teoría ética clásica; mejorar las habilidades para el razonamiento moral; estudiar los sistemas deontológicos (códigos, consejos, etc.); preparar a los alumnos para la vida profesional; y evaluar la actuación de los medios de comunicación. Además se revisan los resultados de una investigación llevada a cabo en 14 países miembros de la UE. En concreto, analizamos las respuestas dadas por 50 profesores de la asignatura a un cuestionario en el que, entre otras cosas, fueron preguntados sobre los objetivos pedagógicos que persiguen al impartir la asignatura.

PALABRAS CLAVE: Ética de la Comunicación - Enseñanza - Educación Superior Profesionalismo - Razonamiento moral

\footnotetext{
${ }^{1}$ Autor correspondiente:

Jesús Díaz del Campo Lozano: Profesor asociado de la Facultad de Empresa y Comunicación y de la Facultad de Derecho de la Universidad Internacional de La Rioja (UNIR). Miembro del Grupo de Investigación Comunicación y Sociedad Digital. España.

Correo: jesus.diaz@unir.net
} 


\title{
PEDAGOGICAL GOALS IN THE TEACHING OF COMMUNICATION ETHICS
}

\begin{abstract}
Which are the main pedagogical goals a Communication Ethics professor must try to reach? In this paper we analyze the most relevant answers the literature on the subject gives to this question: Fostering moral reasoning skills; surveying current ethical practice; preparing students for professional work; sistematically evaluating media performance; and understanding classical ethical theory. We also present the main findings of a survey carried out in 14 European Union member states. We analyze the responses of 50 Comnunication Ethics teachers who have been asked to fill in a questionnaire about the pedagogical goals they try to reach when teaching their subject.
\end{abstract}

KEY WORDS: Communication Ethics - Teaching - Higher education Professionalism - Moral reasoning

\section{INTRODUCCIÓN}

"Cualquier discusión sobre las posibles metas en la enseñanza de la ética debe comenzar por considerar un número importante de enormes dificultades". Estas palabras de Callahan (1980, p. 61) son fiel reflejo de la especial naturaleza de la Ética, que la diferencia significativamente de otras disciplinas como la Historia o el Derecho, por poner dos ejemplos. Esta naturaleza especial se acentúa en el caso de los programas de Ética Profesional o Aplicada, como la Ética de la Comunicación, y se deriva, entre otras cosas, del hecho de que por un lado, está constituida, como cualquier otra disciplina, por un cuerpo de conocimientos, métodos y conceptos clave; pero, por otro, trata cuestiones relativas a los valores, el carácter moral o el "buen” comportamiento. Gran parte de las confusiones y problemas que puede entrañar la enseñanza de la asignatura se derivan de esta dicotomía.

En consecuencia, no es difícil extraer la gran diversidad de planteamientos, incluso podría decirse en último término que casi tantos como profesores de la asignatura. De hecho, la bibliografía no hace sino crear nuevas interrogantes más que contestar las existentes. Resulta sumamente complicado inclinarse por un enfoque y señalarlo como el ideal, como puede comprobarse examinando los numerosos estudios sobre este asunto llevados a cabo en Estados Unidos y en Europa (Black, 1992; Braun, 1999; Callahan y Bok, 1980; Christians, 1978; Christians y Covert, 1980; Christians y Lambeth, 1996; Christians, Rotzoll y Fackler, 1998; Elliot, 1984; The Hastings Center, 1980; Lambeth, Christians y Cole, 1994; Payne, 1992; Peck, 1991; Reis, 2000; Thomas, 2000; Van Horn, 1997). 
No obstante, dentro de esa diversidad, es posible entresacar una serie de metas básicas, comunes en los estudios de diversos autores, y aplicables, con ligeras variantes, a todos los cursos de Ética de la Comunicación. Dicho curso, conforme a las particularidades ya examinadas, debería buscar un punto medio entre los dos extremos que constituyen un planteamiento excesivamente descriptivo y un abuso de la ética normativa. Es decir, ni la mera exposición de casos prácticos, explicando la manera en que los profesionales de la comunicación actúan usualmente y la forma en que deberían hacerlo; ni la afirmación tajante de unas reglas o unos planteamientos totalmente absolutos y válidos para cualquier situación suponen el enfoque más adecuado para un curso que debe buscar el equilibrio entre ambas posturas. Un punto de encuentro que confluye en la llamada ética reflexiva, que pasa, entre otras cosas, por buscar el razonamiento y el análisis crítico por parte de los alumnos.

Ese enfoque se concreta, en el caso del profesional de la comunicación, en una serie de objetivos pedagógicos muy claros, establecidos en uno de los estudios ya mencionados (Lambeth, Christians y Cole, 1994), y que han sido tomados como referencia en todos los trabajos posteriores:

- Entender la teoría ética clásica

- Mejorar las habilidades para el razonamiento moral

- Estudiar los sistemas deontológicos (códigos, consejos, etc.)

- Preparar a los alumnos para la vida profesional

- Evaluar la actuación de los medios de comunicación

A continuación, y antes de explicar la metodología y el analizar los resultados del estudio llevado a cabo en 14 países de la Unión Europea con esos cinco objetivos pedagógicos como base, se explicará brevemente la naturaleza de cada uno de ellos.

\subsection{Entender la teoría ética clásica.}

La Ética está presente en todos los ámbitos de la vida, y el mundo de la comunicación no es una excepción. Más en concreto, existen conexiones entre las teorías éticas fundamentales y muchas áreas de la Deontología de la Comunicación, como por ejemplo el respeto a la verdad o, la intimidad y la vida privada. Por ello, resulta útil cambiar el enfoque más habitual y "en lugar de centrarse en la rutina diaria del periodismo, detenerse en la filosofía y en los fundamentos teóricos en los que se basa dicha rutina diaria" (Merrill, 1997, preface).

Sin embargo, este objetivo suele ser siempre uno de los menos valorados por los alumnos, que lo consideran poco atractivo, algo que puede atribuirse "a su escasísima formación filosófica" (Barroso, 1985, p. 11-12). A pesar de ello, son muchos los autores que resaltan la necesidad de incluir esta parte en el programa, ya que, de otro modo, los alumnos "no entenderían la deontología si no recibieran antes unos conocimientos y corrientes éticas" (Barroso, 1985, p. 11-12). De hecho, el estudio de casos prácticos, probablemente el método de enseñanza más apropiado para la asignatura, resulta 
inútil "si no viene precedido por el estudio en profundidad de los vínculos existentes con las doctrinas éticas más importantes" (Bivins, 1993, p. 4).

En consecuencia, el profesor debe intentar establecer con la mayor claridad posible la relación entre estas teorías y el mundo de la comunicación, porque, teniendo en cuenta el escepticismo inicial de los alumnos, si no lo hace así, este objetivo estará condenado irremisiblemente al fracaso. En este sentido, es recomendable que, a pesar de tratarse de algunos de los pensadores más importantes de la historia, el profesor no omita sus limitaciones, "hay que enseñar a los alumnos que la obediencia ciega a cualquier filosofía nunca es suficiente si queremos realizar análisis serio de una cuestión moral" (Bivins, 1993, p.6).

Otra limitación de carácter práctico para la consecución de este objetivo viene marcada por el tiempo, y la imposibilidad material de explicar todas las teorías éticas fundamentales que han surgido a lo largo de la historia, lo que lleva a la necesidad de elegir entre unas y otras. Examinando las propuestas de diversos autores (Barroso, 1985; Bivins, 1993; Black, 1992, Christians y Covert, 1980; Christians, Rotzoll y Fackler, 1998; Lambeth, Christians y Cole, 1994; Merrill, 1997, Peck, 2001; Suárez Villegas, 2001), hay una serie de pensadores y teorías comunes a la gran mayoría de ellas. En particular, la ética clásica griega (especialmente Aristóteles); el deontologismo (Kant); el utilitarismo (Bentham y Mill); la ética discursiva (Habermas); la teoría de la justicia de Rawls; y, en menor medida, la ética cristiana y las teorías del desarrollo moral, son las perspectivas más recomendadas por la bibliografía.

No obstante, es obvio que ninguna de estas teorías proporciona una solución para la totalidad de problemas éticos que se le pueden presentar al profesional. Por ello, es conveniente no presentar las distintas posiciones como opciones excluyentes, sino más bien "como soluciones integradoras" (Suárez Villegas, 2001, p. 14). Merrill (1997, cap. 10), por su parte, habla de "mutualismo ético", consistente en que el profesional puede encontrar algo útil en cada una de las teorías éticas, y el objetivo final sería formar un sistema filosófico propio, personal, como síntesis de todas las anteriores, resaltando en cada caso por qué y en qué aspectos concretos pueden resultar de utilidad.

\subsection{Mejorar las habilidades para el razonamiento moral.}

De la misma manera que el mundo de la comunicación no constituye una excepción y cuenta con una serie de modelos éticos, también los dilemas morales a los que hace frente el profesional de la comunicación deben ser resueltos a través de un proceso de razonamiento moral. Es éste el momento del proceso educativo donde el peligro del adoctrinamiento está más latente que nunca, por lo que el profesor ha de ser extremadamente cuidadoso, y huir, en la medida de lo posible, de los consejos ad hoc, esto es, su misión será enseñar a los alumnos a pensar por sí mismos, más que decirles qué pensar. Es decir, hay que buscar "una enseñanza activa, que promueva la capacidad de reflexión y de interpretación del alumno, estimulando su "operar", esto es, su actividad autoformativa y su actividad asimiladora de conocimientos" (Vázquez, 1980, p. 12). 
Por lo demás, este objetivo guarda una estrecha relación con las teorías del desarrollo moral de autores como Kohlberg, Piaget o Gilligan², y busca llevar a los alumnos hacia los estadios más avanzados dentro del proceso evolutivo y de desarrollo de su carácter moral. Asimismo, está íntimamente conectado con el conocimiento de las teorías éticas, que constituyen, para muchos autores, el instrumento ideal para el razonamiento y la toma de decisiones. Así por ejemplo, Day y Butler (1989) hablan de tres fases que corresponderían con la explicación de los principios de deontología de la comunicación más importantes; el desarrollo de las teorías éticas; y finalmente, la aplicación de las segundas a ejemplos de situaciones en los que los primeros entren en juego. Sobre todo, resaltan que "la enseñanza del razonamiento moral debería reconocer los lazos entre las disciplinas del periodismo y la filosofia".

\subsection{Estudiar los sistemas deontológicos (códigos, consejos, etc.)}

La autorregulación constituye uno de los indicadores más fieles del grado de desarrollo ético de una actividad, es signo de actuación responsable y en última instancia supone, o debe suponer, la suma de las conciencias individuales de los que ejercen esa actividad. Por tanto, la enseñanza de los distintos mecanismos deontológicos (códigos, consejos, etc.) que formalizan materialmente de la autorregulación parece obligada. De hecho, en sentido inverso, es evidente que el grado de autorregulación de una actividad determina en gran medida cómo se enseña esa actividad. En este sentido, la proliferación de estos mecanismos en los últimos años sería otro punto a favor de su inclusión en el temario. Más concluyente aún son los Principios de Ética Profesional del Periodismo promulgados por la UNESCO en 1983, cuyo apartado octavo dice textualmente: "Incumbe al periodista, por ética profesional, el conocer las disposiciones existentes sobre este tema y que están contenidas en las convenciones internacionales, declaraciones y resoluciones". Esta declaración, por sí sola, convertiría en una exigencia, cuanto menos moral, la enseñanza de todos estos mecanismos.

Ahora bien, el contrapunto necesario viene por el modo en que se expliquen. Los códigos, los consejos de prensa, y en general las disposiciones deontológicas tienen sus limitaciones, por lo que no es conveniente ofrecer una visión demasiado idílica de los mismos, ni tampoco centrarse únicamente en sus puntos fuertes y ventajas, que son muchos (Johannesen, 1988, p. 59-64).

Ni los códigos ni la autorregulación en general son una varita mágica que acabe de raíz con todos los problemas de una actividad, pero sí que suponen una herramienta

\footnotetext{
2 Explicar en detalle teorías sobrepasaría los objetivos de este trabajo. No obstante, en su aplicación al mundo de la comunicación pueden verse, entre otros, Elliott, D. (1991). Moral Development Theories and the Teaching of Ethics, en Journalism Educator, 46, (3), p. 18-24; Goree, K. (2000). Teaching Moral Development in Journalism Education, en Journal of Mass Media Ethics, 15, (2), p. 101-114; o Tucker, E. M. y Stout, D.A. (1999). Teaching Ethics: The Moral Development of Educators, en Journal of Mass Media Ethics, 14, (2), p. 107-118.
} 
útil, que hay que examinar en todas sus dimensiones, incluidas las menos positivas, a las que se llegará antes o después. Mejor será hacerlo antes de ejercer como profesional, ya que de este modo, el alumno asumirá otro aspecto esencial de la autorregulación y, en especial, de los códigos: que son documentos en constante renovación y mejora. Por ello conviene no limitarse a los contenidos del código, sino analizar también el trasfondo histórico, social y cultural.

\subsection{Evaluar la actuación de los medios de comunicación.}

Este cuarto objetivo constituye una primera aplicación concreta de la reflexión ética a la realidad de la actividad profesional concreta, en este caso, el periodismo y/o la comunicación. Por otro lado, ayuda a que los alumnos no conciban el curso como un mero ejercicio abstracto y comprueben que el armazón teórico que el profesor les suministra tiene una utilidad clara y que incluso resulta necesario.

Evaluar la actuación de los medios de comunicación no es, por otro lado, una meta en absoluto alejada de la naturaleza de la Ética, que estudia el comportamiento del ser humano y, en este caso concreto, la buena o mala práctica profesional. En este sentido, lo que en última instancia busca este objetivo es hacer conscientes a los alumnos de lo importante que es la Ética, de las graves consecuencias que puede acarrear el no tenerla presente a la hora de actuar.

Sin embargo, la idea de que la resolución de los dilemas éticos es algo personal, y que sólo uno mismo es el responsable de sus actuaciones queda muy en entredicho en el mundo de la comunicación, debido a la autonomía tan limitada de la que goza el profesional. El resto de factores que intervienen en el proceso, como la audiencia o la empresa de comunicación, entre otros, deben ser muy tenidos en cuenta. Es decir, la actuación del profesional no ha de ser examinada desde la esfera meramente individual sino ir más allá, $\mathrm{y}$, en concreto, tratar de definir su rol o función en la sociedad (Elliot, 1984; Thomas, 2000). En este sentido, ya hace muchos años se ponía de manifiesto la relación entre ambas esferas, dado que "la ética trata con la conducta, y la conducta es un problema de relaciones sociales" (Church, 1939, 172).

Definir el papel del profesional de la comunicación en la sociedad nos llevará, irremediablemente, a definir sus responsabilidades frente a los distintos colectivos antes aludidos. Solo así será posible juzgar en su verdadera dimensión la actuación de los medios. Para ello puede utilizarse, a modo de baremo, los sistemas deontológicos, los principios que aparezcan en códigos, los pronunciamientos de los consejos de prensa, e incluso la teoría y los conceptos éticos.

\subsection{Preparar a los alumnos para la vida profesional.}

Sin duda, el aspecto más importante de la Ética es su dimensión práctica, la aplicación de los principios a la acción. La traslación de esta máxima al ámbito de la formación del profesional conllevaría que la aspiración última e ineludible sería formar buenos profesionales con un gran sentido de la ética. Frente al objetivo anterior que miraba al 
ámbito social, el énfasis pasa a la esfera individual, a pesar de esa autonomía limitada, pero que existe en cierto grado, al fin y al cabo. Es el ideal al que no hay que renunciar, que el profesional de la comunicación tome las mejores decisiones posibles. Distinto será que pueda llevarlas a efecto o no, en función de la intervención del resto de afectados.

La siguiente cuestión sería plantearse en qué consiste exactamente formar a buenos profesionales desde el punto de vista de la Ética. La respuesta, en el caso de cualquier ética aplicada, y mucho más en la comunicación, conduce irremediablemente al concepto de responsabilidad. Si en las asignaturas de carácter más práctico el alumno aprende las técnicas precisas para desarrollar su actividad; en el caso de la Ética y Deontología se trataría más bien de hacerle ver que su actividad tiene una incidencia directa en el buen funcionamiento de las sociedades democráticas, por lo que su responsabilidad es enorme. Por otro lado, la dimensión ética es inherente la actividad comunicativa, es un rasgo necesario para poder hablar de profesionalismo y, además, trasciende a todo el acto comunicativo. Por ello, su importancia debe ser puesta de manifiesto ya que, sin ella, no puede hablarse, de ninguna manera, de un buen profesional.

Todo ello conlleva, como es fácil imaginar, que se dote a la asignatura de un componente eminentemente práctico, por ejercitar las habilidades para decidir de la manera más ética posible. Al fin y al cabo se trata de unas capacidades que se perfeccionan con la práctica diaria. Por lo tanto, aunque la viabilidad de este objetivo ha sido puesta en duda (Barrer y Elliot, 2000, p. 24-25), parece razonable pensar que es posible alcanzar una actitud más ética, al menos en algunos alumnos, lo que ya será motivo suficiente para sentirse satisfecho.

En definitiva, y como conclusión a este apartado, la aspiración última del profesor no debe ser otra que la de conseguir que los alumnos lleguen a pensar por sí mismo, que cuenten con las herramientas necesarias para poder hacer frente a los complejos dilemas éticos que surgirán en el ejercicio de su actividad, y que tomen las mejores decisiones posibles. Además, los objetivos señalados son complementarios y nada excluyentes, y a veces convergen, para su consecución, en varias de las ideas y métodos básicos. Al fin y al cabo, el buen profesional del periodismo será alguien que tome buenas decisiones desde el punto de vista ético, ejecutando sus habilidades para el razonamiento moral, y apoyándose tanto en las teorías filosóficas como en los valores propios de su actividad, y en especial en los instrumentos de autorregulación.

\section{METODOLOGÍA}

Una vez examinadas las diversas posiciones teóricas, a continuación se presentan los resultados de una investigación realizada con el objetivo principal de conocer la realidad de la asignatura en los centros de educación superior de 14 países miembros de la Unión Europea (España, Portugal, Francia, Italia, Grecia, Gran Bretaña, Irlanda, Holanda, Bélgica, Alemania, Austria, Suecia, Finlandia y Dinamarca). De esta manera, 
se recogen las opiniones de 50 profesores encargados en impartir la asignatura en los países mencionados.

Se trata de una muestra suficientemente representativa, por cuanto, entre otras cosas, aúna una gran diversidad de perspectivas y modelos pedagógicos, derivados de circunstancias y variables como el sexo, la edad, la formación previa o el tipo de institución académica en el que se desempeñan las labores profesionales. En cuanto a la distribución de la muestra por países, es la siguiente: España (ocho profesores); Portugal (siete); Francia (cuatro); Italia (cuatro); Grecia (uno); Alemania (cuatro); Austria (dos); Holanda (cuatro); Bélgica (tres); Reino Unido (cinco); Irlanda (uno); Suecia (dos); Finlandia (tres); y Dinamarca (dos).

Se trata además de una investigación muy novedosa, por cuanto ni sobre la enseñanza de la asignatura en general ni sobre los objetivos pedagógicos que debe perseguir en particular existen apenas referencias bibliográficas fuera de las ya mencionadas en Estados Unidos. En la Unión Europea, los intentos de analizar la enseñanza de la Ética de la Comunicación en este ámbito geográfico se refieren a un número limitado de países (Thomas, 2000) o constituyen más bien un manual para impartir la asignatura (Sonnenberg y Thomas, 1996).

Los objetivos pedagógicos incluidos en el cuestionario son los analizados anteriormente, seleccionados a partir del estudio de Lambeth, Christians y Cole (1994). Por otro lado, partiendo de la suposición de que, posiblemente, los cinco, o la mayoría de ellos, formarán parte de la relación de metas planteadas por los profesores encargados de impartir la asignatura se han establecido una serie de categorías para poder delimitar con más precisión cuáles de estos objetivos son los más valorados y establecer una especie de escala de prioridades. En concreto, los profesores han especificado si consideran cada uno de estos objetivos "imprescindible", "muy importante", "importante", "poco importante", o bien "nada importante" o "prescindible".

\section{ANÁLISIS Y DISCUSIÓN}

Los datos globales (Tabla 1) muestran una clara preeminencia de tres objetivos por encima del resto. Mejorar las capacidades para el razonamiento moral, estudiar los sistemas deontológicos y preparar a los alumnos para la vida profesional son las tres metas más valoradas por los encuestados, algo que atestigua el hecho de que más de un $60 \%$ de ellos los considere imprescindibles o muy importantes.

Tabla 1. Valoración de objetivos pedagógicos.

\begin{tabular}{|l|c|c|c|c|c|}
\hline & $\begin{array}{c}\text { Razonam. } \\
\text { moral }\end{array}$ & $\begin{array}{c}\text { Estudio sists. } \\
\text { deontológicos }\end{array}$ & $\begin{array}{c}\text { Preparac. } \\
\text { vida prof. }\end{array}$ & $\begin{array}{c}\text { Evaluación } \\
\text { medios }\end{array}$ & $\begin{array}{c}\text { Teoría } \\
\text { ética }\end{array}$ \\
\hline Imprescindible & 34,0 & 24,0 & 32,0 & 20,0 & 8,0 \\
\hline Muy importante & 30,0 & 36,0 & 32,0 & 38,0 & 6,0 \\
\hline
\end{tabular}


Ética de la Comunicación - Enseñanza - Educación Superior - Profesionalismo Razonamiento moral

\begin{tabular}{|l|c|c|c|c|c|}
\hline Importante & 26,0 & 32,0 & 24,0 & 22,0 & 36,0 \\
\hline Poco importante & 8,0 & 4,0 & 4,0 & 10,0 & 34,0 \\
\hline Nada im. & 2,0 & 4,0 & 8,0 & 10,0 & 16,0 \\
\hline
\end{tabular}

Fuente: Elaboración propia

Los otros dos objetivos son menos valorados, aunque existe una sensible diferencia entre los resultados de uno y de otro. Así, la evaluación de los medios de comunicación es una meta casi tan esencial como las anteriores, ya que el porcentaje de encuestados que lo consideran, cuanto menos, importante, se sitúa en torno al $80 \%$. Por su parte, el entendimiento de la teoría ética clásica es claramente el objetivo menos valorado, ya que la mitad de los encuestados lo considera poco o nada importante.

En este sentido, hay que señalar que, como ya se indicó antes, parte de los expertos apuesta por apoyarse en los principales autores y teorías filosóficas como criterio básico para enseñar al alumno a tomar decisiones éticas, algo que, a tenor de los resultados, no parece muy común entre los profesores europeos. Algunos de ellos se han referido de manera expresa a este hecho en una de las últimas preguntas del cuestionario, en la que se les ofrecía la posibilidad de proponer medidas para mejorar la enseñanza de la asignatura. Una de las respuestas más frecuentes en este punto fue que el limitado número de horas provoca, entre otras cosas, que haya que seleccionar mucho los contenidos del curso, punto en el que la teoría ética clásica suele quedar, a menudo, relegada.

Del mismo modo, en comparación con las opiniones mostradas por los profesores estadounidenses en los diversos estudios llevados a cabo, los resultados guardan varias similitudes, ya que también en ese caso el razonamiento moral era el objetivo más valorado, pues un $75 \%$ de los profesores lo consideraba indispensable; y la teoría ética clásica era, con diferencia, el que recibía una menor importancia, si bien con mejores resultados que en los países analizados ahora, hasta el punto de que un 50\% de los profesores valoraba este objetivo como "importante" y un 18,3\% como "indispensable". Tomando como referencia una y otra encuesta, las discrepancias más significativas entre los profesores estadounidenses y los europeos se centran en el estudio de los sistemas deontológicos, y la preparación de los alumnos de la vida profesional, ya que, frente a lo que ocurre en Europa, en el estudio de Lambeth, Christians y Cole (1994) el primero de ellos se consideraba más importante que el segundo.

Por lo que respecta a los resultados por sexos, una primera lectura es que las mujeres son más rotundas en sus valoraciones mientras que los varones se muestran más moderados. Así, las profesoras se decantan de una manera más clara por el razonamiento moral y la preparación para la vida profesional, porque un $46,7 \%$ de ellas considera uno y otro como objetivos imprescindibles, frente a un $28,6 \%$ y un $25,7 \%$ de los hombres, respectivamente. Además, ninguna de ellas los considera prescindibles mientras que ese resultado sí que obtiene un porcentaje, aunque residual, entre los varones. 
Tabla 2.1. Valoración de objetivos pedagógicos. Hombres.

\begin{tabular}{|l|c|c|c|c|c|}
\hline & $\begin{array}{c}\text { Razonam. } \\
\text { moral }\end{array}$ & $\begin{array}{c}\text { Estudio sists. } \\
\text { deontológicos }\end{array}$ & $\begin{array}{c}\text { Preparac. } \\
\text { vida prof. }\end{array}$ & $\begin{array}{c}\text { Evaluación } \\
\text { medios }\end{array}$ & $\begin{array}{c}\text { Teoría } \\
\text { ética }\end{array}$ \\
\hline Imprescindible & 28,6 & 22,8 & 25,7 & 20,0 & 8,6 \\
\hline Muy importante & 37,1 & 31,4 & 34,3 & 40,0 & 5,7 \\
\hline Importante & 25,7 & 37,1 & 25,7 & 22,8 & 34,3 \\
\hline Poco importante & 5,7 & 2,8 & 2,8 & 8,6 & 40,0 \\
\hline Nada imp. & 2,8 & 5,7 & 2,8 & 8,6 & 11,4 \\
\hline
\end{tabular}

Fuente: Elaboración propia

Algo parecido ocurre con la teoría ética, pues una cuarta parte de mujeres considera que se trata de un objetivo prescindible, porcentaje que entre los hombres se queda en un $11,4 \%$, no obstante lo cual hay que aclarar que sumando los resultados de esa categoría y la de "poco importante", la valoración global realizada por ambos sexos es similar. Los otros dos objetivos pedagógicos, el estudio de sistemas deontológicos, y la evaluación de la actuación de los medios de comunicación obtienen, por el contrario, resultados muy similares entre los hombres y las mujeres.

Tabla 2.1. Valoración de objetivos pedagógicos. Mujeres.

\begin{tabular}{|l|c|c|c|c|c|}
\hline & $\begin{array}{c}\text { Razonam. } \\
\text { moral }\end{array}$ & $\begin{array}{c}\text { Estudio sists. } \\
\text { deontológicos }\end{array}$ & $\begin{array}{c}\text { Preparac. } \\
\text { vida prof. }\end{array}$ & $\begin{array}{c}\text { Evaluación } \\
\text { medios }\end{array}$ & $\begin{array}{c}\text { Teoría } \\
\text { ética }\end{array}$ \\
\hline Imprescindible & 46,7 & 26,7 & 46,7 & 20,0 & 6,7 \\
\hline Muy imp. & 13,3 & 46,7 & 26,7 & 33,3 & 13,3 \\
\hline Importante & 26,7 & 20,0 & 20,0 & 20,0 & 40,0 \\
\hline Poco importante & 13,3 & 6,7 & 6,7 & 13,3 & 13,3 \\
\hline Nada imp. & 0 & 0 & 0 & 13,3 & 26,7 \\
\hline
\end{tabular}

Fuente: Elaboración propia

En todo caso, los datos de uno y otro sexo no hacen sino corroborar la unanimidad en la clasificación de las diferentes metas y en ese sentido, la gran atención que recibe la mejora de las capacidades del razonamiento moral demuestra que, con independencia del país, del tipo de institución educativa, o de otras circunstancias, la toma de decisiones éticas es considerada como un elemento fundamental de la dimensión deontológico de la actividad periodística y es la faceta que, desde esta asignatura, más interesa perfeccionar tanto como sea posible durante el proceso formativo del futuro profesional de la información.

En cuanto al análisis por países, hay que comenzar aclarando que, por un lado, se han distribuido los 14 países mencionados en cuatro grandes zonas geográficas: Sur de Europa (España, Portugal, Francia, Italia y Grecia); Europa Central (Alemania, Austria, Holanda y Bélgica), Norte de Europa (Suecia, Finlandia y Dinamarca) e Islas (Reino Unido e Irlanda). Del mismo modo, en aras de alcanzar una mayor claridad, se ha elaborado una tabla independiente para cada uno de los objetivos, para que la 
comparación entre las distintas zonas, y, en concreto, la importancia que le otorgan los profesores de cada una de ellas, sea más fácil de examinar.

Tabla 3.1. Valoración objetivos. Mejorar habilidades para razonamiento moral.

\begin{tabular}{|l|c|c|c|c|}
\hline & Sur & Europa Central & Islas & Norte \\
\hline Imprescindible & 29,2 & 23,3 & 60,0 & 42,8 \\
\hline Muy imp. & 33,3 & 30,7 & 40,0 & 14,3 \\
\hline Importante & 33,3 & 23,0 & 0 & 28,6 \\
\hline Poco impo. & 4,2 & 15,4 & 0 & 14,3 \\
\hline Nada imp. & 0 & 7,7 & 0 & 0 \\
\hline
\end{tabular}

Fuente: Elaboración propia

En primer lugar, el razonamiento moral, que es el objetivo más valorado en los resultados globales, obtiene sus mejores resultados, con diferencia, en el Reino Unido e Irlanda, ya que la totalidad de los profesores de estos países lo considera una meta imprescindible o muy importante. La mecánica tradicional de enseñanza en esta zona, con una constante aplicación de los casos prácticos, e integrado en muchos casos las cuestiones éticas en proyectos de carácter más global referidos a la actividad periodística en su totalidad, conlleva que esta meta tenga tanta importancia.

Tabla 3.2. Valoración objetivos. Estudiar los distintos sistemas deontológicos.

\begin{tabular}{|l|c|c|c|c|}
\hline & Sur & Europa Central & Islas & Norte \\
\hline Imprescindible & 16,7 & 23,0 & 50,0 & 28,5 \\
\hline Muy imp. & 29,2 & 53,8 & 33,3 & 28,5 \\
\hline Importante & 45,8 & 15,4 & 16,7 & 28,5 \\
\hline Poco imp. & 4,2 & 0 & 0 & 14,3 \\
\hline Nada imp. & 4,2 & 7,7 & 0 & 0 \\
\hline
\end{tabular}

Fuente: Elaboración propia

El otro lado de la balanza lo constituyen los países de Europa Central, en los que una cuarta parte de los profesores $(23,1 \%)$ considera este objetivo prescindible o poco importante. Dado que en estos países la preeminencia del enfoque práctico ha sido algo habitual en la enseñanza del periodismo, y que se trata de una zona que cuenta además con varias escuelas profesionales, la explicación de este resultado, opuesto por ejemplo al del Reino Unido e Irlanda, países con los que comparte varios de los elementos pedagógicos fundamentales, puede buscarse en el número de horas de clase más limitado en algunos de los centros, o bien en que la preparación para la vida profesional, que se valora mucho más entre estos profesores, sea entendida, incluso desde el punto de vista de la ética, como un perfeccionamiento de las habilidades más técnicas y rutinarias del periodista.

Los países del Sur también otorgan una gran importancia a este objetivo ya que más del $95 \%$ de encuestados lo sitúa en el tramo entre imprescindible e importante. Dado 
que la cifra de programas de carácter universitario es proporcionalmente más alta que en otras zonas, la realidad viene a demostrar que la enseñanza de la asignatura cuenta con una serie de metas comunes a las que, ni facultades ni escuelas, con independencia de su carácter y de su vocación general, deben renunciar. Finalmente, en el Norte también existe una gran valoración, con un $85,7 \%$ de los profesores que lo considera entre imprescindible e importante. Es una muestra más de la unanimidad general en cuanto a este objetivo, que la gran mayoría de los encuestados considera básico para los alumnos.

Lo mismo ocurre con el estudio de los distintos sistemas deontológicos. Las islas son la zona geográfica que mayor valoración otorga a este objetivo, y vuelven a ser la única en la que el 100\% lo considera, cuanto menos, importante. El gran desarrollo de la autorregulación en Gran Bretaña, que lo ha convertido en el país paradigmático en estos aspectos explica esta gran importancia. En el resto de países se observan unos resultados muy equilibrados, sobre todo entre el Centro y el Norte, mientras que en el Sur la importancia recibida por este objetivo es ligeramente inferior.

La preparación para la vida profesional obtiene sus mejores resultados en las islas, donde el 100\% de los profesores las considera al menos muy importante, algo lógico dado que el aprendizaje ha sido la vía de acceso más frecuente, y que las líneas maestras del propio sistema formativo han sido elaboradas históricamente en torno a esta idea principal. El Norte y Europa Central también consideran este objetivo básico, con resultados muy similares, y de nuevo los profesores del Sur son los que menos lo valoran. $\mathrm{Y}$ es que, en resumen, el estudio de los sistemas deontológicos y la preparación para el desarrollo de la actividad profesional parecen ser objetivos que marchan paralelos y cuya valoración es prácticamente la misma en todos los casos.

Tabla 3.3. Valoración objetivos. Preparar a los alumnos para la vida profesional.

\begin{tabular}{|l|c|c|c|c|}
\hline & Sur & Europa Central & Islas & Norte \\
\hline Imprescindible & 20,8 & 46,1 & 16,7 & 57,1 \\
\hline Muy imp. & 25,0 & 30,7 & 83,3 & 14,3 \\
\hline Importante & 37,5 & 15,4 & 0 & 14,3 \\
\hline Poco imp. & 4,2 & 7,7 & 0 & 0 \\
\hline Nada imp. & 12,5 & 0 & 0 & 14,3 \\
\hline
\end{tabular}

Fuente: Elaboración propia

Muy distinto es el caso de la evaluación de la actuación de los medios de comunicación, objetivo respecto al que las opiniones están muy repartidas, especialmente en el Sur y en el Norte, donde la proporción de profesores que los sitúa en los dos primeros lugares y en los tres últimos de la escala es muy similar. El Reino Unido e Irlanda son nuevamente los lugares con una consideración más alta, en concreto un $83,3 \%$ sumando los dos primeros lugares de la escala de valoración. En el resto de países, dado que los datos están muy repartidos, puede decirse que se trata de una meta importante, pero menos, que las tres anteriores. 
Ética de la Comunicación - Enseñanza - Educación Superior - Profesionalismo Razonamiento moral

Tabla 3.4. Valoración objetivos. Evaluar actuación de medios de comunicación.

\begin{tabular}{|l|c|c|c|c|}
\hline & Sur & Europa Central & Islas & Norte \\
\hline Imprescindible & 16,7 & 15,4 & 33,3 & 28,5 \\
\hline Muy imp. & 33,3 & 53,8 & 50,0 & 14,3 \\
\hline Importante & 29,1 & 15,4 & 0 & 28,5 \\
\hline Poco imp. & 4,2 & 7,7 & 16,7 & 28,5 \\
\hline Nada imp. & 16,7 & 7,7 & 0 & 0 \\
\hline
\end{tabular}

Fuente: Elaboración propia

Finalmente, la teoría ética es una meta especialmente desdeñada en Europa Central. Ninguno de los profesores de esta zona la considera imprescindible o muy importante y el porcentaje que los sitúa en los dos últimos grados se aproxima al 70\%. La tradición de escuelas profesionales en Holanda y Bélgica, y del modelo de aprendizaje en el caso de Alemania y Austria, aunque ya no se mantienen en exclusiva, han provocado que algunas de las ideas básicas de estos enfoques perduren hoy en día, a pesar de que la enseñanza en el ámbito universitario es ya una realidad en los cuatro países citados.

Tabla 3.5. Valoración objetivos. Entender la teoría ética clásica.

\begin{tabular}{|l|c|c|c|c|}
\hline & Sur & Europa Central & Islas & Norte \\
\hline Imprescindible & 8,3 & 0 & 16,7 & 14,3 \\
\hline Muy imp. & 8,3 & 0 & 16,7 & 14,3 \\
\hline Importante & 45,8 & 30,7 & 16,7 & 28,5 \\
\hline Poco imp. & 20,8 & 61,5 & 33,3 & 14,3 \\
\hline Nada imp. & 16,7 & 7,7 & 16,7 & 14,3 \\
\hline
\end{tabular}

Fuente: Elaboración propia

En el resto de zonas hay una coincidencia en que la teoría ética es siempre la menos valorada, aunque hay gran diversidad, con un equilibrio en todos los grados de la escala, quizá correspondiente a que cada profesor, en función de la institución y de su formación, la valora de una manera u otra. De todos modos, es el objetivo respecto al que existen más dudas y el dato más sorprendente puede ser la importancia que recibe en Escandinavia. Es sorprendente por doble motivo, porque en muchos de los centros, la asignatura es Ética y Derecho de la Información, y, por otro lado, porque Dinamarca y Suecia cuentan con varias escuelas profesionales. Quizá esto explica la diversidad, ya que la distribución de opiniones sobre este objetivo en esta área es totalmente simétrica, es decir, la valoración media sería importante, lo que constituye, por otro lado, un mejor resultado que en otras zonas.

A modo de resumen final se podría decir que si bien la naturaleza de la institución educativa (Universidad, formación profesional, etc.) determina en cierta medida la consideración de unas $\mathrm{u}$ otras metas pedagógicas, existe unanimidad con independencia de las diversas variables en valorar el perfeccionamiento de las capacidades para el razonamiento moral como la meta fundamental de cualquier 
curso de Ética de la Comunicación, pero sin olvidarse de otros objetivos también muy importantes, como, por este orden, el estudio de los sistemas deontológicos, la preparación para la vida profesional, y la evaluación de los medios de comunicación. La teoría ética clásica recibe una importancia secundaria, y mucho más variable, en función de las características de la profesión periodística en el país concreto, y de otras circunstancias diversas.

\section{CONCLUSIONES}

Los resultados de la investigación ponen de manifiesto que la metodología docente de la asignatura más usual es la que se basa en un proceso dialógico, expresión de una concepción ética reflexiva, que supone un punto medio entre los planteamientos meramente descriptivos y los excesivamente normativos. De este modo, la siempre deseable meta de la participación activa del alumnado será mucho más asequible.

En este sentido, las habilidades para la toma de decisiones éticas y, en concreto, el ejercicio del razonamiento moral, y la preparación para la vida profesional son los objetivos pedagógicos unánimemente más valorados por los profesores de la asignatura. En el caso del primero, tanto los principios deontológicos como la propia teoría ética pueden constituir criterios válidos para decidir, proceso en el cual el "cómo" ha de ser más importante que el "qué", esto es, el mecanismo empleado por el alumno es lo verdaderamente fundamental, por encima de su decisión concreta final. La preparación para el futuro ejercicio profesional constituye una meta igualmente loable, incluso en el ámbito universitario, ya que supone el reconocimiento de esa orientación eminentemente práctica que impregna la Ética, como ya pusiera de manifiesto Aristóteles.

En un segundo plano de importancia se sitúa el estudio de los sistemas deontológicos, objetivo respecto al cual resulta especialmente adecuado no limitarse a estudiar sus aspectos positivos, sino analizar igualmente las objeciones realizadas desde diversos sectores, sus puntos menos favorables, y el proceso que ha llevado a su elaboración, ya que al fin y al cabo estos mecanismos y documentos han de ser instrumentos en continua renovación.

Finalmente, cabe decir que en todo caso la efectividad de este tipo de asignaturas será muy difícil de determinar, y en concreto será complicado establecer si los alumnos que pasan por ellos actúan más éticamente, aunque sí es razonable suponer que sean más conscientes y estén mejor preparados frente a las diversas problemáticas morales.

\section{REFERENCIAS}

Barger, W. y Elliot, D. (2000). "Journalism ethics classes: Do they make better journalists?" En Quill, 88, (7), p. 24-25.

Barroso, P. (1985). Fundamentos deontológicos de las Ciencias de la Información. Barcelona: Mitre. 
Bivins, T. H. (1993). A worksheet for ethics instruction and exercises in reason. En Journalism Educator, 48, (2), p. 4-16.

Black, J. (1992). “Media Ethics”. En Murray, M. D. y Ferri, A. J. (eds.). Teaching Mass Communication. A Guide to Better Instruction (pp. 235-255). New York: Praeger.

Braun, M. J. (1999). “Media Ethics Education: A Comparison of Student Responses". En Journal of Mass Media Ethics, 14, (3), p. 171-182.

Callahan, D. (1980). "The goals in the teaching of ethics". En Callahan, D. y Bok, S. (eds.) Ethics Teaching in Higher Education, (pp.61-80). The Hastings Center Series in Ethics. New York: Plenum Press.

Callahan, D. y Bok, S. (eds.) (1980). Ethics Teaching in Higher Education. New York: Plenum Press.

Christians, C. G. (1978). "Variety of Approaches Used in Teaching Media Ethics". En Journalism Educator, 1, (1), p. 3-8, 24.

Christians, C. G. y Covert, C. L. (1980). Teaching Ethics in Journalism Education. Hastings-on-Hudson, New York: The Hastings Center.

Christians, C. G. y Lambeth, E. (1996). “The Status of Ethics Instruction in Communication Departments". En Communication Education. 45, (3), p. 236-244.

Christians, C. G.; Rotzoll, K. B. y Fackler, M. (1998). Media Ethics: Cases and Moral Reasoning. White Plains, New York: Longman.

Church, G. F. (1939). “Analysis of Social Relationships in Teaching Journalistic Ethics”. En Journalism Quarterly, 16, (2), p. 172-174.

Day, L. A. y Butler, J. M. (1989). The Teaching of Ethics and Moral Reasoning in Scholastic Journalism: The Pedagogical Imperative. Paper presented at the midwinter Meeting of the Association for Education in Journalism and Mass Communication, Secondary Education Division. St. Petersburg, FL. Manuscrito mecanografiado.

Elliot, D. T. (1984). Toward the Development of a Model for Journalism Ethics Instruction. Ph. D. dissertation, Faculty of the Graduate School of Education, Harvard University.

The Hastings Center (1980). The Teaching of Ethics in Higher Education. New York: The Hastings Center.

Johannesen, R. L. (1988). "What Should We Teach about Formal Codes of Communication Ethics". En Journal of Mass Media Ethics, 2, (1), p. 59-64. 
Lambeth, E.; Christians, C. y Cole, K. (1994). "Role of the Media Ethics Course in the Education of Journalists". En Journalism Educator, 49, (3), p. 20-26.

Merrill, J. C. (1997). Journalism Ethics: Philosophical Foundations for News Media. New York: St. Martin's.

Payne, N. (1992). Teaching the "Unteachable". Toward a model for ethics instruction in Canadian university schools of journalism. Ph. D. dissertation. Ottawa, Ontario: Faculty of Graduate Studies and Research, Carleton University,

Peck, A. L. (2001). Foolproof or Foolhardy? Presenting Ethical Theory to Journalism Students. Ph. D. dissertation. Ohio University Department of Philosophy and College of Arts and Sciences.

Reis, R. (2000). Teaching Media Ethics in a Multicultural Setting. En Journal of Media Ethics, 15, (3), p. 194-205.

Sonnenberg, U. y Thomas, B. (1996). Journalistic decision-taking in Europe case by case. Maastricht: European Journalism Centre.

Suárez, J. C. (2001). Principios de ética profesional: a propósito de la actividad informativa. Madrid: Tecnos.

Thomas, B. (2000). How Journalism Ethics is Taught Around Europe: Three Examples. En Pattyn, B. (ed.). Media Ethics Opening Social Dialogue (pp. 375-389). Lovaina: Peeters.

Vázquez, F. (1980). Ética. Planteamientos y horizontes actuales. Zaragoza: Luis Vives.

Van Horn, T. L. (1997). Teaching Ethics in Communication Courses. PH. D. dissertation. Fullerton: Faculty of California State University.

\section{Jesús Díaz del Campo Lozano}

Profesor de Comunicación Oral y Escrita y de Ética y Deontología de la Comunicación en la Universidad Internacional de La Rioja (UNIR), España. Doctor en Periodismo por la Universidad Complutense de Madrid, España. Miembro del Grupo de Investigación Comunicación y Sociedad Digital, de la Universidad Internacional de La Rioja, España. Profesionalmente ha ejercido como periodista en diversos medios e instituciones durante más de 15 años. 